\begin{tabular}{|c|l|}
\hline Title & On Implicit Second-Order Ordinary Differential Equations: Completely Integrable and Clairaut Type \\
\hline Author(s) & Takahashi, M. \\
\hline Citation & $\begin{array}{l}\text { Journal of Dynamical and Control Systems, 13(2), 273-288 } \\
\text { https://doi.org/10.1007/310883-007-9013-9 }\end{array}$ \\
\hline Issue Date & 2007-04 \\
\hline Doc URL & http://hdl.handle.net/2115/24429 \\
\hline Rights & The original publication is available at www.springerlink.com \\
\hline Type & article (author version) \\
\hline File Information & JDCS13-2.pdf \\
\hline
\end{tabular}

Instructions for use 


\title{
On implicit second order ordinary differential equations: Completely integrable and Clairaut type
}

\author{
Masatomo Takahashi \\ Department of Mathematics, Hokkaido University, \\ Sapporo 060-0810, JAPAN \\ e-mail: takahashi@math.sci.hokudai.ac.jp
}

August 15, 2006

\begin{abstract}
We study the implicit second order ordinary differential equations with complete integral. In this paper, we give a characterization of the implicit second order ordinary differential equations with smooth complete integral which we call Clairaut type equations. Besides, we consider properties of the Clairaut type equations and present the duality among special completely integrable equations with respect to Engel-Legendre transformations.
\end{abstract}

\section{Introduction}

The classical Clairaut equation (ALEXIS CLAUDE CLAIRAUT [2], 1713-1765) is one of the typical examples of the first order ordinary differential equations which is given by

$$
y=x p+f(p)
$$

where $p=d y / d x$ and $f$ is a smooth function. It is treated as an example of a non-linear equation which is easily solved. Moreover, it has a beautiful geometric structure as follows: There exists a "general solution" that consists of line $y=t \cdot x+f(t)$ where $t$ is a parameter and the singular solution is the envelope of that family. The Clairaut type equation was investigated by Dara [4] as a generalization of the classical Clairaut equation. In [8], it has been given a characterization of first order Clairaut type equations. Also in $[9,16]$, they have studied a generic classification of Clairaut type equations for the case of first order differential equations.

In this paper, we shall discuss Clairaut type equations for the case of second order ordinary differential equations. For implicit second order ordinary differential equations, we consider two second order ordinary classical Clairaut equations. Indeed, in [11, 12, 15, 18] higher order

2000 Mathematics Subject classification: 34A09, 34A26

Key Words and Phrases. implicit second order ordinary differential equation, completely integrable, Clairaut type equation, Engel-Legendre transformation 
classical Clairaut equations were studied. In particular, one of second order ordinary classical Clairaut equations is defined by

$$
y=x p-\frac{1}{2} x^{2} q+f(q)
$$

where $p=d y / d x, q=d^{2} y / d x^{2}$ and $f$ is a smooth function. The "general solution" is formed by replacing the derivatives by arbitrary constants (cf. [18]):

$$
y=s \cdot x+\frac{1}{2} r \cdot x^{2}+f(r)
$$

for $r, s \in \mathbb{R}$.

On the other hand, the second order ordinary classical Clairaut equation was studied by Shyuichi Izumiya (cf. [1]). Consider the following equation similar to the first order classical Clairaut equation:

$$
p=x q+f(q)
$$

where $p=d y / d x, q=d^{2} y / d x^{2}$ and $f$ is a smooth function. We can easily solve this equation and "general solution" is given by

$$
y=\frac{1}{2} r \cdot x^{2}+f(r) \cdot x+s
$$

for $r, s \in \mathbb{R}$, in detail see Example 2.4. We will refer such classical equations to second order Clairaut type equations (see, §3). In $\S 2$, we introduce the notions of smooth complete solutions, complete solutions and Engel-Legendre transformations. In $\S 3$, we give a characterization of second order Clairaut type equations (Theorem 3.1). As a property of second order Clairaut type equations, we give a relationship between second order classical Clairaut equations and Clairaut type equations (Theorem 3.4). In order to prove Theorem 3.4, we use the Engel-Legendre transformations. In $\S 4$, we define a special complete solution and establish the principle of duality among implicit second order ordinary differential equations with special complete integral. We show that if $F=0$ is specially completely integrable, then $F=0$ is of Clairaut type (Corollary 4.2). Finally, we determine the normal forms of special complete solutions (Theorem 4.3).

All manifolds and map germs considered here are differentiable of class $C^{\infty}$, unless stated otherwise.

\section{Basic notions}

We consider an implicit second order ordinary differential equation of the form

$$
F\left(x, y, \frac{d y}{d x}, \frac{d^{2} y}{d x^{2}}\right)=0
$$

where $F$ is a smooth function. In this paper, we assume that $F:\left(J^{2}(\mathbb{R}, \mathbb{R}),\left(x_{0}, y_{0}, p_{0}, q_{0}\right)\right) \longrightarrow$ $(\mathbb{R}, 0)$ is a germ of a submersion, then $S=F^{-1}(0)$ is a 3-dimensional submanifold germ of $J^{2}(\mathbb{R}, \mathbb{R})$ near $\left(x_{0}, y_{0}, p_{0}, q_{0}\right)$. Here $J^{2}(\mathbb{R}, \mathbb{R})$ is the 2-jet bundle of 1 -variable function which may be considered as $\mathbb{R}^{4}$ with coordinates given by $(x, y, p, q)$. Consider the canonical Engel structure $\xi$ on $J^{2}(\mathbb{R}, \mathbb{R})$ which is given by the following pair of 1 -forms as the kernel,

$$
\alpha_{1}=d y-p d x, \alpha_{2}=d p-q d x .
$$


We now define the notion of solutions. A smooth solution of $F=0$ (or, a classical solution) is a function germ $y=f(x)$ at the origin such that $\left(0, f(0), f^{\prime}(0), f^{\prime \prime}(0)\right)=\left(x_{0}, y_{0}, p_{0}, q_{0}\right)$ and $F\left(x, f(x), f^{\prime}(x), f^{\prime \prime}(x)\right)=0$. In other words, there exists a function germ $f:(\mathbb{R}, 0) \longrightarrow \mathbb{R}$ such that the image of 2 -jet extension of $f, j^{2} f:(\mathbb{R}, 0) \longrightarrow J^{2}(\mathbb{R}, \mathbb{R})$, is contained in $F^{-1}(0)$. Obviously, the map germ $j^{2} f$ is an Engel immersion germ. Here we say that a map germ $\gamma$ : $(\mathbb{R}, 0) \longrightarrow J^{2}(\mathbb{R}, \mathbb{R})$ is an Engel immerison germ if $\gamma$ is an immerison germ and $\gamma^{*} \alpha_{1}=\gamma^{*} \alpha_{2}=0$. Also a geometric solution of $F=0$ is a smooth immersion germ $\gamma:(\mathbb{R}, 0) \longrightarrow F^{-1}(0)$ such that $\gamma^{*} \alpha_{1}=\gamma^{*} \alpha_{2}=0$, namely, $\gamma$ is an Engel immersion germ and the image of $\gamma$ is contained in $F^{-1}(0)$. If we denote $\gamma(t)=(x(t), y(t), p(t), q(t))$ in the coordinates on $J^{2}(\mathbb{R}, \mathbb{R})$, then the conditions of an Engel immersion germ are equivalent to $\gamma^{\prime}(t) \neq 0, y^{\prime}(t)=p(t) x^{\prime}(t)$ and $p^{\prime}(t)=q(t) x^{\prime}(t)$. It is easy to prove the following lemma:

Lemma 2.1 Let $\gamma:(\mathbb{R}, 0) \longrightarrow F^{-1}(0)$ be a geometric solution. Suppose that $x^{\prime}(0) \neq 0$. Then there exist a diffeomorphism germ $\phi:(\mathbb{R}, 0) \longrightarrow(\mathbb{R}, 0)$ and a function germ $f$ such that $\gamma \circ \phi(t)=\left(t, f(t), f^{\prime}(t), f^{\prime \prime}(t)\right)$.

According to the above result, we may define the notion of singular point of solutions. We say that $t_{0}$ is a geometric singular point of the geometric solution $\gamma$ if $x^{\prime}\left(t_{0}\right)=0$. Thus, the solution is multi-valued around the geometric singular point. It is clear that $t_{0}$ is a geometric singular point of $\gamma$ if and only if $\left(x^{\prime}\left(t_{0}\right), y^{\prime}\left(t_{0}\right), p^{\prime}\left(t_{0}\right)\right)=(0,0,0)$ or $\left(x^{\prime}\left(t_{0}\right), y^{\prime}\left(t_{0}\right)\right)=(0,0)$.

We call $\left(x_{0}, y_{0}, p_{0}, q_{0}\right)$ a $\pi_{1}$-singular point or a $\pi$-singular point of $F=0$ if $F=F_{q}=0$ at $\left(x_{0}, y_{0}, p_{0}, q_{0}\right)$ or $F=F_{p}=F_{q}=0$ at $\left(x_{0}, y_{0}, p_{0}, q_{0}\right)$, where $\pi_{1}(x, y, p, q)=(x, y, p)$ and $\pi(x, y, p, q)=(x, y)$. We denote by $\Sigma_{\pi_{1}}(F)$ and $\Sigma_{\pi}(F)$ the sets of $\pi_{1}$-singular points and $\pi$ singular points respectively. We also call the set $D_{F}=\pi\left(\Sigma_{\pi}(F)\right)$ a discriminant set of $F=0$.

In $[5,13,14]$, it has been investigated the existence of geometric solutions and a local classification of generic second order ordinary differential equations. In this paper, we are interested in the following notions (cf. $[3,6,7,9,16,17])$ :

By the definition of smoothness of the solution (i.e., classical solution) and a parametrized version of lemma 2.1, a smooth complete solution of $F=0$ is defined by a two-parameter family of smooth function germs $y=f(r, s, t)$ such that

$$
F\left(t, f(r, s, t), \frac{\partial f}{\partial t}(r, s, t), \frac{\partial^{2} f}{\partial t^{2}}(r, s, t)\right)=0
$$

and the germ $j_{*}^{2} f:\left(\mathbb{R}^{2} \times \mathbb{R}, 0\right) \longrightarrow F^{-1}(0) \subset J^{2}(\mathbb{R}, \mathbb{R})$ defined by

$$
j_{*}^{2} f(r, s, t)=\left(t, f(r, s, t), \frac{\partial f}{\partial t}(r, s, t), \frac{\partial^{2} f}{\partial t^{2}}(r, s, t)\right)
$$

is an immersion. We remark that $j_{*}^{2} f$ is an immersion germ if and only if

$$
\operatorname{rank}\left(\begin{array}{lll}
(\partial f / \partial r) & \left(\partial^{2} f / \partial r \partial t\right) & \left(\partial^{3} f / \partial r \partial t^{2}\right) \\
(\partial f / \partial s) & \left(\partial^{2} f / \partial s \partial t\right) & \left(\partial^{3} f / \partial s \partial t^{2}\right)
\end{array}\right)(0)=2 .
$$

On the other hand, we consider the corresponding definition of a parametrized version for geometric solutions. Let $\Gamma:\left(\mathbb{R}^{2} \times \mathbb{R}, 0\right) \longrightarrow\left(F^{-1}(0),\left(x_{0}, y_{0}, p_{0}, q_{0}\right)\right)$ be a two-parameter family of geometric solutions of $F=0$. We call $\Gamma$ a complete solution at $\left(x_{0}, y_{0}, p_{0}, q_{0}\right)$ if

$$
\operatorname{rank}\left(\begin{array}{cccc}
(\partial x / \partial r) & (\partial y / \partial r) & (\partial p / \partial r) & (\partial q / \partial r) \\
(\partial x / \partial s) & (\partial y / \partial s) & (\partial p / \partial s) & (\partial q / \partial s) \\
(\partial x / \partial t) & (\partial y / \partial t) & (\partial p / \partial t) & (\partial q / \partial t)
\end{array}\right)(0)=3
$$


where $\Gamma(r, s, t)=(x(r, s, t), y(r, s, t), p(r, s, t), q(r, s, t))$. It follows that $\Gamma$ is a diffeomorphism germ.

We say that an equation $F=0$ is completely integrable at $\left(x_{0}, y_{0}, p_{0}, q_{0}\right)$ if there exists a complete solution of $F=0$ at $\left(x_{0}, y_{0}, p_{0}, q_{0}\right)$. The uniqueness of the complete solution is given by the following proposition:

Proposition 2.2 Let $\Gamma_{1}$ and $\Gamma_{2}:\left(\mathbb{R}^{2} \times \mathbb{R}, 0\right) \longrightarrow\left(F^{-1}(0),\left(x_{0}, y_{0}, p_{0}, q_{0}\right)\right)$ be complete solutions of $F=0$ at $\left(x_{0}, y_{0}, p_{0}, q_{0}\right)$. Then there exists a diffeomorphism germ $\Phi:\left(\mathbb{R}^{2} \times \mathbb{R}, 0\right) \longrightarrow$ $\left(\mathbb{R}^{2} \times \mathbb{R}, 0\right)$ of the form $\Phi(r, s, t)=\left(\phi_{1}(r, s), \phi_{2}(r, s, t)\right)$ such that $\Gamma_{1} \circ \Phi=\Gamma_{2}$.

Proof. Suppose that the assertion does not hold. Since the solution is a two-parameter family of curves in $F^{-1}(0)$, then there exists a point $\left(x_{1}, y_{1}, p_{1}, q_{1}\right) \in\left(F^{-1}(0),\left(x_{0}, y_{0}, p_{0}, q_{0}\right)\right)$ such that $\Gamma_{1\left(r_{1}, s_{1}\right)}$ and $\Gamma_{2\left(r_{2}, s_{2}\right)}$ are transversal near the point $\left(x_{1}, y_{1}, p_{1}, q_{1}\right)$. Then we can construct a germ $\Gamma:\left(\mathbb{R}^{2} \times \mathbb{R}, 0\right) \longrightarrow\left(F^{-1}(0),\left(x_{1}, y_{1}, p_{1}, q_{1}\right)\right)$ such that (at least) $\Gamma_{t}$ is an immersion germ,

$$
\frac{\partial y}{\partial r}(r, s, t)=p(r, s, t) \frac{\partial x}{\partial r}(r, s, t), \frac{\partial p}{\partial r}(r, s, t)=q(r, s, t) \frac{\partial x}{\partial r}(r, s, t)
$$

and

$$
\frac{\partial y}{\partial s}(r, s, t)=p(r, s, t) \frac{\partial x}{\partial s}(r, s, t), \frac{\partial p}{\partial s}(r, s, t)=q(r, s, t) \frac{\partial x}{\partial s}(r, s, t),
$$

where $\Gamma(r, s, t)=(x(r, s, t), y(r, s, t), p(r, s, t), q(r, s, t))$. If we calculate second order partial derivatives of above equalities, then we get

$$
\frac{\partial^{2} p}{\partial s \partial r}=\frac{\partial q}{\partial s} \cdot \frac{\partial x}{\partial r}+q \cdot \frac{\partial^{2} x}{\partial s \partial r} \text { and } \frac{\partial^{2} p}{\partial r \partial s}=\frac{\partial q}{\partial r} \cdot \frac{\partial x}{\partial s}+q \cdot \frac{\partial^{2} x}{\partial r \partial s} .
$$

Therefore we obtain the equality $(\partial q / \partial s) \cdot(\partial x / \partial r)=(\partial q / \partial r) \cdot(\partial x / \partial s)$. This contradicts the fact that $\Gamma_{t}$ is an immersion germ.

Let an equation $F=0$ be completely integrable at $\left(x_{0}, y_{0}, p_{0}, q_{0}\right)$. We say that a geometric solution $\gamma:\left(\mathbb{R}, t_{0}\right) \longrightarrow\left(F^{-1}(0),\left(x_{0}, y_{0}, p_{0}, q_{0}\right)\right)$ is a singular solution of $F=0$ around $\left(x_{0}, y_{0}, p_{0}, q_{0}\right)$ if for any representative $\widetilde{\gamma}: U \longrightarrow F^{-1}(0)$ of $\gamma$, where $U$ is an open subset around $t_{0}$, and any open subinterval $(c, d) \subset U,\left.\widetilde{\gamma}\right|_{(c, d)}$ is never contained in a leaf of a complete solution (cf. $[7,10])$.

Now we define contact singular points, complete singular solutions and second order contact singular points (see [1]).

Around points $z \in S=F^{-1}(0)$ such that the contact plane $\xi_{z}$ intersects $T_{z} S$ transversally, it is easy to see that a complete solution exists by integrating the line field $\xi \cap T S$. We call points where transversality fails to hold contact singular points and denote by $\Sigma_{c}=\Sigma_{c}(F)$ the set of contact singular points. It is easy to check that the set of contact singular points is given by

$$
\Sigma_{c}(F)=\left\{z \in J^{2}(\mathbb{R}, \mathbb{R}) \mid F(z)=0, F_{x}(z)+p F_{y}(z)+q F_{p}(z)=0, F_{q}(z)=0\right\} .
$$

From the definition of singular solutions, it is easy to see that a geometric solution $\gamma:\left(\mathbb{R}, t_{0}\right) \longrightarrow$ $\left(S,\left(x_{0}, y_{0}, p_{0}, q_{0}\right)\right)$ is a singular solution only if it is contained in $\Sigma_{c}(F)$. We will also consider the subset $\Delta=\Delta(F) \subset \Sigma_{c}$ which is defined to be the set of points $z \in \Sigma_{c}$ such that $T_{z} S$ coincides with the kernel of $\alpha_{1}(z)$. Explicitly, it is given by $\Delta=\left\{z \in \Sigma_{c} \mid F_{p}(z)=0\right\}$.

We now assume that $\Sigma_{c} \neq \emptyset$ and $\Sigma_{c}$ is a 2-dimensional submanifold. Then we call a diffeomorphism, if it exists,

$$
\Phi:\left(\mathbb{R} \times \mathbb{R},\left(0, t_{0}\right)\right) \longrightarrow\left(\Sigma_{c},\left(x_{0}, y_{0}, p_{0}, q_{0}\right)\right)
$$


such that for each $a \in(\mathbb{R}, 0), \Phi(a, \cdot):\left(\mathbb{R}, t_{0}\right) \longrightarrow\left(\Sigma_{c},\left(x_{0}, y_{0}, p_{0}, q_{0}\right)\right)$ is a singular solution, a complete singular solution around $\left(x_{0}, y_{0}, p_{0}, q_{0}\right)$.

Also, around points $z \in \Sigma_{c}$ such that $\xi_{z}$ intersects $T_{z} \Sigma_{c}$ transversally in $T_{z} S$, it is easy to see that a complete singular solution exists by integrating the line field $\xi \cap T \Sigma_{c}$ if it is not contained in the complete solution. Around points where transversality does not hold a complete singular solution need not exist. We call such points second order contact singular points and denote by $\Sigma_{c c}=\Sigma_{c c}(F)$ the set of second order contact singular points.

In [1], were given the existence conditions of complete solution and complete singular solution under a regularity condition.

Theorem 2.3 [1, Theorems 1.1, 1.3 and Lemma 3.7] Suppose that 0 is a regular value of $\left.F_{q}\right|_{S}$.

(1) $F=0$ is completely integrable at $z_{0}$ if and only if $z_{0} \notin \Sigma_{c}$ or $\Sigma_{c}$ is a 2-dimensional manifold around $z_{0}$.

(2) $F=0$ is completely integrable and $\Sigma_{c} \neq \emptyset$. Then $F=0$ admits a complete singular solution around $z_{0} \in \Sigma_{c}$ if and only if $z_{0} \notin \Sigma_{c c}$ or $\Sigma_{c c}$ is a 1-dimensional manifold around $z_{0}$.

(3) $F=0$ is completely integrable. Then $\Sigma_{c c}$ is contained in $\Delta$.

Here we give examples illustrating the notions of complete solutions and complete singular solutions.

Example 2.4 (Second order ordinary classical Clairaut equations)

(i) Let $F=-y+x p-(1 / 2) x^{2} q+q^{2}\left(f(q)=q^{2}\right)$. In this case $F_{x}+p F_{y}+q F_{p} \equiv 0, F_{q}=$ $-(1 / 2) x^{2}+2 q$. Therefore the contact singular set is given by

$$
\Sigma_{c}=\left\{(x, y, p, q) \mid y=x p-(1 / 16) x^{4}, q=(1 / 4) x^{2}\right\} .
$$

By Theorem 2.3, this equation is completely integrable. In fact, differentiation of the equation with respect to $x$ yields

$$
\left(2 q-(1 / 2) x^{2}\right)(d q / d x)=0 .
$$

By the vanishing of the second factor, we obtain $q=r$ and $p=r x+s$ where $r, s \in \mathbb{R}$. Substitution of these values into $F=0$ leads to

$$
y=(1 / 2) r x^{2}+s x+r^{2}
$$

for each $r, s \in \mathbb{R}$. This is a general solution which gives rise to the complete solution $\Gamma$ : $\mathbb{R}^{2} \times \mathbb{R} \longrightarrow S$,

$$
\Gamma(r, s, t)=\left(t,(1 / 2) r t^{2}+s t+r^{2}, r t+s, r\right) .
$$

Also observe that the map

$$
\Phi: \mathbb{R} \times \mathbb{R} \longrightarrow \Sigma_{c}
$$

given by

$$
\Phi(a, t)=\left(t, \frac{1}{48} t^{4}+a t, \frac{1}{12} t^{3}+a, \frac{1}{4} t^{2}\right)
$$

is a complete singular solution. In this case, $\Sigma_{c c}=\emptyset$ and $\Delta=\{(x, y, p, q) \mid x=y=q=0\}$.

(ii) Let $F(x, y, p, q)=-p+q x+f(q)$. In this case $F_{x}+p F_{y}+q F_{p} \equiv 0, F_{q}=x+f^{\prime}(q)$. Thus the contact singular set is given by

$$
\Sigma_{c}=\left\{(x, y, p, q) \mid x=-f^{\prime}(q), p=-q f^{\prime}(q)+f(q)\right\} .
$$


By Theorem 2.3, this equation is also completely integrable. In fact, differentiation of the equation with respect to $x$ yields

$$
\left(x+f^{\prime}(q)\right)(d q / d x)=0 .
$$

By the vanishing of the second factor, we obtain $q=r$ and $p=r x+f(r)$ where $r \in \mathbb{R}$. Substitution of these values into $F=0$ leads to

$$
y=(1 / 2) r x^{2}+f(r) x+s
$$

for each $r, s \in \mathbb{R}$. This is a general solution which gives rise to the complete solution $\Gamma$ : $\mathbb{R}^{2} \times \mathbb{R} \longrightarrow S$,

$$
\Gamma(r, s, t)=\left(t,(1 / 2) r t^{2}+f(r) t+s, r t+f(r), r\right) .
$$

Also observe that the map

$$
\Phi: \mathbb{R} \times \mathbb{R} \longrightarrow \Sigma_{c}
$$

given by

$$
\Phi(a, t)=\left(-f^{\prime}(t), \int\left(t f^{\prime}(t) f^{\prime \prime}(t)-f(t) f^{\prime \prime}(t)\right) d t+a,-t f^{\prime}(t)+f(t), t\right)
$$

is a complete singular solution. In this case, $\Sigma_{c c}=\Delta=\emptyset$.

We consider a transformation of $\mathbb{R}^{4}$. Let $(X, Y, P, Q)$ be another coordinate system on $\mathbb{R}^{4}$ given by $X=q, Y=p-q x, P=y-p x+(1 / 2) q x^{2}, Q=x$. We call the smooth mapping $E: \mathbb{R}^{4} \longrightarrow \mathbb{R}^{4}$

$$
E(x, y, p, q)=\left(q, p-q x, y-p x+(1 / 2) q x^{2}, x\right)
$$

an Engel-Legendre transformation (or, briefly, $E$ - $L$ transformation). By definition, we have $E^{-1}(X, Y, P, Q)=\left(Q, P+Y Q+(1 / 2) X Q^{2}, Y+X Q, X\right)$. If we apply the $E$ - $L$ transformation to our equation $F=0$, we obtain a new equation and call such equation a dual of equation,

$$
F^{*}(X, Y, P, Q)=F\left(Q, P+Y Q+(1 / 2) X Q^{2}, Y+X Q, X\right)
$$

in the coordinate system $(X, Y, P, Q)$.

We calculate partial derivatives at the point $\left(X_{0}, Y_{0}, P_{0}, Q_{0}\right)$ corresponding to $\left(x_{0}, y_{0}, p_{0}, q_{0}\right)$, we can show the following:

$$
\begin{aligned}
& F_{Q}^{*}\left(X_{0}, Y_{0}, P_{0}, Q_{0}\right)=\left(F_{x}+p F_{y}+q F_{p}\right)\left(x_{0}, y_{0}, p_{0}, q_{0}\right) \\
& F_{P}^{*}\left(X_{0}, Y_{0}, P_{0}, Q_{0}\right)=F_{y}\left(x_{0}, y_{0}, p_{0}, q_{0}\right) \\
& F_{Y}^{*}\left(X_{0}, Y_{0}, P_{0}, Q_{0}\right)=\left(x F_{y}+F_{p}\right)\left(x_{0}, y_{0}, p_{0}, q_{0}\right) \\
& F_{X}^{*}\left(X_{0}, Y_{0}, P_{0}, Q_{0}\right)=\left((1 / 2) x^{2} F_{y}+x F_{p}+F_{q}\right)\left(x_{0}, y_{0}, p_{0}, q_{0}\right) .
\end{aligned}
$$

\section{$3 \quad$ Second order Clairaut type equations}

In this section, we give a definition and a characterization of second order Clairaut type equations. The notion of second order Clairaut type equations is one of generalizations of the notion of the second order classical Clairaut equations. 
We say that an equation $F=0$ is a second order Clairaut type equation (a Clairaut type equation for short) at $\left(x_{0}, y_{0}, p_{0}, q_{0}\right)$ if there exist smooth function germs $A(x, y, p, q)$ and $B(x, y, p, q)$ at $\left(x_{0}, y_{0}, p_{0}, q_{0}\right)$ such that

$$
F_{x}+p \cdot F_{y}+q \cdot F_{p}=A \cdot F+B \cdot F_{q}
$$

By definition, if $F=0$ is a Clairaut type equation at $\left(x_{0}, y_{0}, p_{0}, q_{0}\right)$, then $\Sigma_{c}(F)=\Sigma_{\pi_{1}}(F)$ and $\Delta(F)=\Sigma_{\pi}(F)$. Both of classical Clairaut equations are Clairaut type equations, because we may take $A=B=0$ by direct calculations. We will discuss a relationship between the classical Clairaut equations and the Clairaut type equations (cf. Theorem 3.4).

If an equation $F=0$ is of Clairaut type at $\left(x_{0}, y_{0}, p_{0}, q_{0}\right)$, then $F=0$ is completely integrable at $\left(x_{0}, y_{0}, p_{0}, q_{0}\right)$ by $[1$, Lemma 3.1].

The following theorem gives a characterization of the Clairaut type equations in detail.

Theorem 3.1 For an equation $F=0$, the following conditions are equivalent.

(1) $F=0$ is a Clairaut type equation at $\left(x_{0}, y_{0}, p_{0}, q_{0}\right)$.

(2) $F=0$ has a smooth complete solution at $\left(x_{0}, y_{0}, p_{0}, q_{0}\right)$.

Moreover, if $F=0$ satisfies the above condition and $\Sigma_{\pi}(F) \neq 0$, then the discriminant set $D_{F}$ is the envelope of the family of graphs of the complete solution.

Proof. Assume that $F=0$ is a Clairaut type equation at $\left(x_{0}, y_{0}, p_{0}, q_{0}\right)$, then there exist function germs $A$ and $B$ at $\left(x_{0}, y_{0}, p_{0}, q_{0}\right)$ such that $F_{x}+p \cdot F_{y}+q \cdot F_{p}=A \cdot F+B \cdot F_{q}$. We consider a vector field germ $V=\partial / \partial x+p \cdot(\partial / \partial y)+q \cdot(\partial / \partial p)-B \cdot(\partial / \partial q)$ at $\left(x_{0}, y_{0}, p_{0}, q_{0}\right)$. Let $c(t)$ be an integral curve of $V$ such that $c(0) \in F^{-1}(0)$. Then we can calculate that $\left.(d F(c(t)) / d t)\right|_{t=0}=F_{x}+p \cdot F_{y}+q \cdot F_{p}-B \cdot F_{q}=0$. It follows that $V$ is tangent to $F^{-1}(0)$. If we denote $c(t)=(x(t), y(t), p(t), q(t))$, then we have $x^{\prime}(t)=1, y^{\prime}(t)=p(c(t)), p^{\prime}(t)=q(c(t))$ and $q^{\prime}(t)=-B(c(t))$. These equalities guarantee that $c(t)$ is a smooth solution of $F=0$. Then the flow of the vector field $V$ gives the smooth complete solution of $F=0$.

On the other hand, let $y=f(r, s, t)$ be the smooth complete solution of $F=0$ at $\left(x_{0}, y_{0}, p_{0}, q_{0}\right)$. If we calculate the partial derivate of $F\left(t, f(r, s, t), f_{t}(r, s, t), f_{t t}(r, s, t)\right)=0$ with respect to $t$, then we have $F_{x}+f_{t} \cdot F_{y}+f_{t t} \cdot F_{p}+f_{t t t} \cdot F_{q}=0$ at $\left(t, f(r, s, t), f_{t}(r, s, t), f_{t t}(r, s, t)\right) \in$ $F^{-1}(0)$. Since the map $j_{*}^{2} f$ is an immersion germ, then there exists a function germ $B(x, y, p, q)$ at $\left(x_{0}, y_{0}, p_{0}, q_{0}\right)$ such that $B \circ j_{*}^{2} f(r, s, t)=f_{t t t}(r, s, t)$. For any $(x, y, p, q) \in F^{-1}(0)$, there exists $(r, s, t)$ such that $\left(t, f(r, s, t), f_{t}(r, s, t), f_{t t}(r, s, t)\right)=(x, y, p, q)$. Hence we have $F_{x}+p \cdot F_{y}+q \cdot F_{p}=$ $-B \cdot F_{q}$. Since $d F \neq 0$, the above equality means that there exists a function germ $A(x, y, p, q)$ at $\left(x_{0}, y_{0}, p_{0}, q_{0}\right)$ such that $F_{x}+p \cdot F_{y}+q \cdot F_{p}=A \cdot F-B \cdot F_{q}$. This completes the proof by renaming $-B$ as $B$.

For the proof of the second part, we may assume that there exists a smooth complete solution $y=f(r, s, t)$ of $F=0$ around $\left(x_{0}, y_{0}, p_{0}, q_{0}\right)$. By definition, $j_{*}^{2} f(r, s, t) \in \Sigma_{\pi}(F)$ if and only if

$$
\operatorname{rank}\left(\begin{array}{cc}
1 & f_{t} \\
0 & f_{r} \\
0 & f_{s}
\end{array}\right)<2 .
$$

This is equivalent to $f_{r}=f_{s}=0$. Then the set $\Sigma_{\pi}(F)$ is given by the equation $f_{r}=f_{s}=0$ near $\left(x_{0}, y_{0}, p_{0}, q_{0}\right)$. We now consider the family of graphs of the smooth complete solutions which is given by the equation $f(r, s, t)-y=0$ in the $(t, y)$-plane. Then the set $\{(t, f(r, s, t)) \mid$ there exists $(r, s)$ such that $\left.f_{r}(r, s, t)=f_{s}(r, s, t)=0\right\}$ is the envelope of this family by the definition. 
This set is equal to the discriminant set $D_{F}$ by the previous argument. This completes the proof of Theorem 3.1.

We now consider properties of the second order Clairaut type equations. The following proposition asserts that the class of Clairaut type equations is more general than the class of equations satisfying the assumptions of theorem 2.3 , namely, $F=0$ is completely integrable, 0 is a regular value of $\left.F_{q}\right|_{S}$ and $\Sigma_{c} \neq \emptyset$.

Proposition 3.2 Let $F=0$ be completely integrable at $z_{0}, 0$ be a regular value of $\left.F_{q}\right|_{S}$ and $z_{0} \in \Sigma_{c}$. Then $F=0$ is a Clairaut type equation at $z_{0}$.

Proof. By Theorem 2.3, definitions of $\Sigma_{c}$ and $\Sigma_{\pi_{1}}$ (see $\left.\S 2\right), \Sigma_{c}$ and $\Sigma_{\pi_{1}}$ are 2-dimensional submanifolds of $S=F^{-1}(0)$ around $z_{0}$. Since $d F_{q}\left(z_{0}\right) \neq 0$, there exists a function germ $B(x, y, p, q)$ at $z_{0}$ such that $F_{x}+p \cdot F_{y}+q \cdot F_{p}=B \cdot F_{q}$ on $F^{-1}(0)$. Furthermore $d F\left(z_{0}\right) \neq 0$, then the above equality means that there exists a function germ $A(x, y, p, q)$ at $z_{0}$ such that $F_{x}+p \cdot F_{y}+q \cdot F_{p}=A \cdot F+B \cdot F_{q}$.

We also have the following proposition (compare with Theorem 2.3):

Proposition 3.3 Suppose that $F=0$ is a Clairaut type equation at $z_{0}$ and $\Sigma_{c}$ is a 2 -dimensional submanifold around $z_{0}$. Then $\Sigma_{c c}$ is contained in $\Delta$.

Proof. Assume that $\Sigma_{c c} \neq \emptyset$ and let $z_{0} \in \Sigma_{c c}$. Since $d F\left(z_{0}\right) \neq 0$ and $z_{0} \in \Sigma_{c}$, either $F_{y}\left(z_{0}\right) \neq 0$ or $F_{p}\left(z_{0}\right) \neq 0$. First suppose that $F_{y}\left(z_{0}\right) \neq 0$. Due to the implicit function theorem, there exists a smooth function germ $f: V \longrightarrow \mathbb{R}$ where $V$ is an open set of $\mathbb{R}^{3}$ such that, in a neighbourhood of $z_{0}$, a point $(x, y, p, q) \in F^{-1}(0)$ if and only if $f(x, p, q)-y=0$. Thus, without loss of generality, we may assume that $F(x, y, p, q)=f(x, p, q)-y$. Let $\phi: V \longrightarrow S$ denote the smooth map $(x, p, q) \longmapsto(x, f(x, p, q), p, q)$. Since $F=0$ is a Clairaut type equation, we have $\Sigma_{c}=\Sigma_{\pi_{1}}$. It follows that $\phi^{-1}\left(\Sigma_{c}\right)=f_{q}^{-1}(0)$. Also there exist smooth function germs $A$ and $B$ at $z_{0}$ such that the following equality holds:

$$
f_{x}-p+q \cdot f_{p}=A \cdot F+B \cdot f_{q}
$$

From the definition of $\Sigma_{c c}$, we have

$$
f_{q x}(v)+q \cdot f_{q p}(v)=0, f_{q q}(v)=0,
$$

where $v=(x, p, q) \in \phi^{-1}\left(\Sigma_{c c}\right)$. On the other hand, differentiating (1) with respect to $q$ gives

$$
f_{x q}+f_{p}+q \cdot f_{p q}=A_{q} \cdot F+A \cdot f_{q}+B_{q} \cdot f_{q}+B \cdot f_{q q} \cdot
$$

For any $z=(x, y, p, q) \in \Sigma_{c c}$, comparing above equalities we obtain that $f_{p}(v)=0$ and hence $z \in \Delta$.

Suppose that $F_{p}\left(z_{0}\right) \neq 0$. Again, due to the implicit function theorem, there exists a smooth function germ $g: U \longrightarrow \mathbb{R}$ where $U$ is an open set of $\mathbb{R}^{3}$ such that, in a neighbourhood of $z_{0}$, a point $(x, y, p, q) \in F^{-1}(0)$ if and only if $g(x, y, q)-p=0$. Hence, without loss of generality, we may assume that $F(x, y, p, q)=g(x, y, q)-p$. Let $\psi: U \longrightarrow S$ denote the smooth map $(x, p, q) \longmapsto(x, y, g(x, y, q), q)$. Then $\psi^{-1}\left(\Sigma_{c}\right)=g_{q}{ }^{-1}(0)$ and there exist smooth function germs $A$ and $B$ at $z_{0}$ such that the following equality holds:

$$
g_{x}+g \cdot g_{y}-q=A \cdot F+B \cdot g_{q}
$$


From the definition of $\Sigma_{c c}$, we have

$$
g_{q x}(u)+g(u) \cdot g_{q y}(u)=0, g_{q q}(u)=0,
$$

where $u=(x, y, q) \in \psi^{-1}\left(\Sigma_{c c}\right)$. Also differentiating (2) with respect to $q$ gives

$$
g_{x q}+g_{q} \cdot g_{y}+g \cdot g_{y q}-1=A_{q} \cdot F+A \cdot g_{q}+B_{q} \cdot g_{q}+B \cdot g_{q q} .
$$

However, for any $z=(x, y, p, q) \in \Sigma_{c c}$, this case cannot occur.

This completes the proof of Proposition 3.3.

Consider the second order ordinary classical Clairaut equations rather than Clairaut type equations. We give a characterization of second order ordinary classical Clairaut equations as follows:

Theorem 3.4 Let $F=0$ be an equation around $z_{0}=\left(x_{0}, y_{0}, p_{0}, q_{0}\right)$.

(A) The following conditions are equivalent:

(1) $F_{y} \neq 0$ at $z_{0}$ and there exist function germs $A, B:\left(J^{2}(\mathbb{R}, \mathbb{R}), z_{0}\right) \longrightarrow \mathbb{R}$ such that

$$
F_{x}+p \cdot F_{y}+q \cdot F_{p}=A \cdot F \text { and } x \cdot F_{y}+F_{p}=B \cdot F .
$$

(2) There exists a function germ $f:\left(\mathbb{R}, q_{0}\right) \longrightarrow \mathbb{R}$ such that

$$
F^{-1}(0)=\left\{(x, y, p, q) \mid y=p x-(1 / 2) q x^{2}+f(q)\right\} .
$$

(B) The following conditions are equivalent:

(1) $F_{p} \neq 0$ at $z_{0}$ and there exist function germs $A, B:\left(J^{2}(\mathbb{R}, \mathbb{R}), z_{0}\right) \longrightarrow \mathbb{R}$ such that

$$
F_{x}+p \cdot F_{y}+q \cdot F_{p}=A \cdot F \text { and } F_{y}=B \cdot F .
$$

(2) There exists a function germ $f:\left(\mathbb{R}, q_{0}\right) \longrightarrow \mathbb{R}$ such that

$$
F^{-1}(0)=\{(x, y, p, q) \mid p=q x+f(q)\} .
$$

Proof. Item $(A)$. Suppose that $F=0$ satisfies the condition (1). Then $F_{y} \neq 0$ at $z_{0}$. By the implicit function theorem, we may assume that there exists a function germ $h(x, p, q)$ on an open subset of $\mathbb{R}^{3}$ such that $F$ has the form $F(x, y, p, q)=h(x, p, q)-y$. We now consider the dual of equation $F^{*}=0$ (see $\left.\S 2\right)$. Then $F^{*}(X, Y, P, Q)=H(X, Y, Q)-P$ where $H(X, Y, Q)=h(Q, Y+X Q, X)-Y Q-(1 / 2) X Q^{2}$. It follows that $F_{Q}^{*}=H_{Q}$ and $F_{Y}^{*}=H_{Y}$ on $F^{*-1}(0)$. Since $F_{Q}^{*}=F_{x}+p \cdot F_{y}+q \cdot F_{p}$ and $F_{Y}^{*}=x \cdot F_{y}+F_{p}$, we have $H_{Q} \equiv 0$ and $H_{Y} \equiv 0$. Therefore we can put $f(X)=H(X, Y, Q)$, then $F^{*}(X, Y, P, Q)=f(X)-P$. Pulling back by the $E-L$ transformation, we have $F^{-1}(0)=\left\{(x, y, p, q) \mid y=p x-(1 / 2) q x^{2}+f(q)\right\}$. The converse of the proof is obtained by a direct calculation.

Item $(B)$. We can prove by similar arguments of item $(A)$. By the assumption of $F_{p} \neq 0$ at $z_{0}$, again due to the implicit function theorem, we may assume that the form of equation is given by $F(x, y, p, q)=k(x, y, q)-p$ for some function germ $k(x, y, q)$ on an open subset of $\mathbb{R}^{3}$. Also since $F_{y} \equiv 0$ on $F^{-1}(0)$, it follows that the equation is given by $F(x, y, p, q)=k(x, q)-p$. Then the dual equation of $F=0$ is $F^{*}(X, Y, P, Q)=K(X, Q)-Y$ where $K(X, Q)=k(Q, X)-X Q$. Since $F_{Q}^{*}=K_{Q}$ on $F^{*-1}(0)$ and $F_{Q}^{*}=F_{x}+p \cdot F_{y}+q \cdot F_{p}$, we have $K_{Q} \equiv 0$. Therefore we can put $f(X)=K(X, Q)$. Pulling back by the $E$ - $L$ transformation, we have $F^{-1}(0)=\{(x, y, p, q) \mid p=$ $q x+f(q)\}$. The converse of the proof is also obtained by a direct calculation. 


\section{Special completely integrable and Engel-Legendre trans- formations}

In this section, we consider a duality of completely integrable equations with respect to EngelLegendre transformations. In order to introduce a duality, we define a special complete solution of equations. Let $\Gamma:\left(\mathbb{R}^{2} \times \mathbb{R}, 0\right) \longrightarrow\left(F^{-1}(0),\left(x_{0}, y_{0}, p_{0}, q_{0}\right)\right)$ be a complete solution of $F=0$ at $\left(x_{0}, y_{0}, p_{0}, q_{0}\right)$. We call $\Gamma$ a special complete solution if $q(r, s, t)=q(r, s)$, where $\Gamma(r, s, t)=$ $(x(r, s, t), y(r, s, t), p(r, s, t), q(r, s, t))$. We say that $F=0$ is a special completely integrable equation at $\left(x_{0}, y_{0}, p_{0}, q_{0}\right)$ if there exists a special complete solution of $F=0$ at $\left(x_{0}, y_{0}, p_{0}, q_{0}\right)$.

Let $F=0$ be a completely integrable and $F^{*}=F \circ E^{-1}$ be a dual equation of $F=0$ (see $\S 2)$. We denote $\Gamma^{*}=E \circ \Gamma$ where $\Gamma:\left(\mathbb{R}^{2} \times \mathbb{R}, 0\right) \longrightarrow F^{-1}(0)$ is a complete solution of $F=0$.

Since the $E$ - $L$ transformation does not preserve the canonical Engel structure, $F^{*}=0$ might not have a complete solution. However, we can prove the following theorem:

Theorem 4.1 Let $\Gamma:\left(\mathbb{R}^{2} \times \mathbb{R}, 0\right) \longrightarrow F^{-1}(0)$ be a complete solution of $F=0$. Then $\Gamma^{*}$ is a complete solution of $F^{*}=0$ if and only if $\Gamma$ is a special complete solution of $F=0$.

Proof. We denote $\Gamma(r, s, t)=(x(r, s, t), y(r, s, t), p(r, s, t), q(r, s, t))$. By definition,

$$
\begin{aligned}
\Gamma^{*}(r, s, t)= & (q(r, s, t), p(r, s, t)-q(r, s, t) x(r, s, t), \\
& \left.y(r, s, t)-p(r, s, t) x(r, s, t)+(1 / 2) q(r, s, t) x^{2}(r, s, t), x(r, s, t)\right) .
\end{aligned}
$$

Since the $E$ - $L$ transformation is a diffeomorphism germ, $\Gamma^{*}$ is also a diffeomorphism germ. We caluculate that $\left(\Gamma_{(r, s)}^{*}\right)^{*} \alpha_{1}=0$ and $\left(\Gamma_{(r, s)}^{*}\right)^{*} \alpha_{2}=0$ for each $(r, s) \in\left(\mathbb{R}^{2}, 0\right)$. We have

$$
\left(\Gamma_{(r, s)}^{*}\right)^{*} \alpha_{1}=-\left(x(r, s, t)+y(r, s, t)-p(r, s, t) x(r, s, t)+\frac{1}{2} q(r, s, t) x^{2}(r, s, t)\right) \frac{\partial q}{\partial t}(r, s, t)
$$

and

$$
\left(\Gamma_{(r, s)}^{*}\right)^{*} \alpha_{2}=\frac{1}{2} x(r, s, t)(x(r, s, t)-2) \frac{\partial q}{\partial t}(r, s, t) .
$$

If we assume that $(\partial q / \partial t)(r, s, t) \neq 0$ around $t_{0} \in(\mathbb{R}, 0)$, then $x(r, s, t) \equiv 0$ or $x(r, s, t) \equiv 2$ around $t_{0}$ by the continuity of $x(r, s, t)$.

For the case $x(r, s, t) \equiv 0$, we get $y(r, s, t) \equiv 0$ around $t_{0}$ by $\left(\Gamma_{(r, s)}^{*}\right)^{*} \alpha_{1}=0$. On the other hand, when $x(r, s, t) \equiv 2$, we also get $2+y(r, s, t)-2 p(r, s, t)+2 q(r, s, t) \equiv 0$ around $t_{0}$ by $\left(\Gamma_{(r, s)}^{*}\right)^{*} \alpha_{1}=0$.

In the both cases, we come to a contradiction with the fact that $\Gamma$ is a diffeomorphism germ. Therefore we have $(\partial q / \partial t)(r, s, t) \equiv 0$ around 0 .

The converse of the proof is obtained by a direct calculation.

Let $F=0$ be a special completely integrable equation at $z_{0}$. According to Theorem 4.1, the dual equation $F^{*}=0$ is also completely integrable at $z_{0}^{*}=E\left(z_{0}\right)$.

Moreover, let $\Gamma:\left(\mathbb{R}^{2} \times \mathbb{R}, 0\right) \longrightarrow F^{-1}(0)$ be a special complete solution of $F=0$. Since $\Gamma$ is complete solution of $F=0, \Gamma_{(r, s)}(t)$ is a geometric solution of $F=0$ for fixed parameters $(r, s) \in\left(\mathbb{R}^{2}, 0\right)$. From the definition of geometric solution, it follows that $(\partial x / \partial t)(0) \neq 0$ where $\Gamma(r, s, t)=(x(r, s, t), y(r, s, t), p(r, s, t), q(r, s))$. Then we can easily show that $F=0$ has a smooth complete solution. As a corollary of Theorem 3.1, we have the following result: 
Corollary 4.2 Suppose that $F=0$ is a special completely integrable equation at $\left(x_{0}, y_{0}, p_{0}, q_{0}\right)$. Then $F=0$ is a Clairaut type equation at $\left(x_{0}, y_{0}, p_{0}, q_{0}\right)$.

Let $F=0$ be a special completely integrable equation at $\left(x_{0}, y_{0}, p_{0}, q_{0}\right)$. By Corollary 4.2 , $F=0$ has a smooth complete solution $\Gamma:\left(\mathbb{R}^{2} \times \mathbb{R}, 0\right) \longrightarrow\left(F^{-1}(0),\left(x_{0}, y_{0}, p_{0}, q_{0}\right)\right)$ which is given by

$$
\Gamma(r, s, t)=\left(t, f(r, s, t), f_{t}(r, s, t), f_{t t}(r, s, t)\right),
$$

where $f$ is a smooth function germ. By the uniqueness of complete solution (Proposition 2.2) and the definition of special complete solution, there exists a smooth function germ $\alpha$ such that $f_{t t}(r, s, t)=\alpha(r, s)$. Hence the smooth function germ $f$ is represented by

$$
f(r, s, t)=\frac{1}{2} \alpha(r, s) t^{2}+\beta(r, s) t+\gamma(r, s),
$$

where $\alpha, \beta$ and $\gamma$ are smooth function germs. As a consequence, we have the normal forms of special complete solutions.

Theorem 4.3 Let $\Gamma:\left(\mathbb{R}^{2} \times \mathbb{R}, 0\right) \longrightarrow\left(F^{-1}(0),\left(x_{0}, y_{0}, p_{0}, q_{0}\right)\right)$ be a special complete solution of $F=0$ at $\left(x_{0}, y_{0}, p_{0}, q_{0}\right)$. Then $\Gamma$ is represented by the form

$$
\Gamma(r, s, t)=\left(t, \frac{1}{2} \alpha(r, s) t^{2}+\beta(r, s) t+\gamma(r, s), \alpha(r, s) t+\beta(r, s), \alpha(r, s)\right),
$$

where $\alpha, \beta$ and $\gamma$ are smooth function germs.

Remark that by definition of the complete solution, $\alpha, \beta$ and $\gamma$ satisfy the condition

$$
\operatorname{rank}\left(\begin{array}{lll}
(\partial \alpha / \partial r) & (\partial \beta / \partial r) & (\partial \gamma / \partial r) \\
(\partial \alpha / \partial s) & (\partial \beta / \partial s) & (\partial \gamma / \partial s)
\end{array}\right)(0)=2
$$

By definition, both of second order classical Clairaut equations are special completely integrable. We can consider the completely integrable dual equations for such equations.

Acknowledgment. A part of this work was done during the author's stay at the University of Valencia. The author would like to thank the Department of Geometry and Topology and especially Professor M. C. Romero Fuster for her kind hospitality. Also the author would like to thank Professor Shyuichi Izumiya for useful advice and constant encouragement. This work was partially supported by Research Fellowship of Japan Society for the Promotion of Science for Young Scientists.

\section{References}

[1] M. Bhupal, On singular solutions of implicit second-order ordinary differential equations, Hokkaido Math. J. 32 (2003), 623-641.

[2] A. C. Clairaut, Solution de plusieurs problems, Histoire de l'Academie Royale de Sciences, Paris (1734), 196-215.

[3] R. Courant and D. Hilbert, Methods of Mathematical Physics II (Wiley, New York, 1962). 
[4] L. Dara, Singularités générique des équations différentielles multiformes, Bol. Soc. Brasil Mat. 6 (1975), 95-128.

[5] A.A. Davydov, Singularities of limiting directions of generic higher order implicit ODEs, Proc. Stekov Inst. Math. 236 (2002), 124-131.

[6] A. Hayakawa, G. Ishikawa, S. Izumiya and K. Yamaguchi, Classification of generic integral diagrams and first order ordinary differential equations, Internat. J. Math. 5 (1994), 447-489.

[7] S. Izumiya, Singular solutions of first-order differential equations, Bull. London Math. Soc. 26 (1994), 69-74.

[8] S. Izumiya, On Clairaut-type equations, Publ. Math. Debrecen 45 (1994), 159-166.

[9] S. Izumiya and Y. Kurokawa, Holonomic systems of Clairaut type, Differential Geom. Appl. 5 (1995), 219-235.

[10] S. Izumiya and J. Yu, How to define singular solutions, Kodai Math. J. 16 (1993), 227-234.

[11] J. D. Kečkić, Clairaut's equations of higher order, Publ. Inst. Math. 12 (26) (1971), 63-66.

[12] J. D. Kečkić, Additions to Kamke's treatise VIII: On singular solutions of generalised Clairaut's equation, Univ. Beograd. Publ. Elektrotehn. Fak. Ser. Mat. Fiz. No. 577-578 (1977), 30-32.

[13] M. Kossowski, Fiber completions, contact singularities and single valued solutions for $C^{\infty}$-second order ODE, Canad. J. Math. 48 (1996), 849-870.

[14] M. Lemasurier, Singularities of second-order implicit differential equations: A geometrical approch, J. Dynam. Control Systems 7 (2001), 277-298.

[15] D. S. Mitrinović and J. D. Kečkić, Variations and generalisations of Clairaut's equations, Univ. Beograd. Publ. Elektrotehn. Fak. Ser. Mat. Fiz. No. 716-734 (1981), 11-21.

[16] M. Takahashi, Bifurcations of ordinary differential equations of Clairaut type, J. Differential Equations 190 (2003), 579-599.

[17] M. Takahashi, Bifurcations of completely integrable first order ordinary differential equations, (Russian) Sovrem. Mat. Prilozh. No.33 (2005), 110-125; translation in J. Math. Sci.

[18] W. H. Witty, Generalization of Clairaut's differential equation, Amer. Math. Monthly 59 (1952), 100-102. 